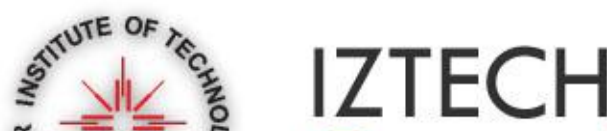

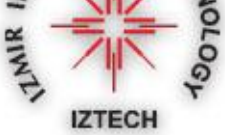 \\ Open Access Articles
}

\section{Controlling Spontaneous Emission of CdSe Nanoparticles Dispersed in Electrospun Fibers of Polycarbonate Urethane}

The IZTECH Faculty has made this article openly available. Please share how this access benefits you. Your story matters.

\begin{tabular}{|l|l|}
\hline Citation & $\begin{array}{l}\text { Demir, M, Soyal, D, Ünlü, C, Kuş, M, and Özçelik, S, } \\
\text { "Controlling Spontaneous Emission of CdSe Nanoparticles } \\
\text { Dispersed in Electrospun Fibers of Polycarbonate Urethane" } \\
\text { Journal of Physical Chemistry C @ 2009 American Chemical } \\
\text { Society Published }\end{array}$ \\
\hline As Published & $10.1021 /$ jp903899s \\
\hline Publisher & American Chemical Society Published \\
\hline Version & PUBLISHED ARTICLE \\
\hline Accessed & FRI JULY 5 15:32:32 GMT 2013 \\
\hline Citable Link & http://hdl.handle.net/11147/ \\
\hline Terms of Use & $\begin{array}{l}\text { Article is made available in accordance with the publisher's } \\
\text { policy and may be subject to Turkish copyright law. Please } \\
\text { refer to the publisher's site for terms of use. }\end{array}$ \\
\hline Detailed Terms & \\
\hline
\end{tabular}




\title{
Controlling Spontaneous Emission of CdSe Nanoparticles Dispersed in Electrospun Fibers of Polycarbonate Urethane
}

\author{
Mustafa M. Demir,* Duygu Soyal, Caner Ünlü, Mahmut Kuş, and Serdar Özçelik* \\ Department of Chemistry, ĺzmir Institute of Technology, Gülbahçe Köyü, Urla 35430 İzmir, Turkey
}

Received: February 23, 2009

\begin{abstract}
Luminescent fibrous composite films consisting of submicrometer diameter fibers were prepared by electrospinning of segmented polycarbonate urethane (PCU) in dimethyl formamide and tri- $n$-octylphosphine oxide (TOPO)-capped CdSe nanocrystals (5 nm in diameter) in toluene. Using a pair of conductive electrodes separated with an air gap, we successfully produced randomly deposited and uniaxially aligned electrospun fibers. The surface structure of the electrospun fibers was studied using atomic force microscopy (AFM) and was compared to the corresponding film prepared by casting. In cast film, tapping mode AFM imaging suggests that hard urethane segments organize into rodlike morphology dispersed in soft polycarbonate. When PCU/ $\mathrm{CdSe}$ dispersions were subjected to electrospinning, copolymer domains were forced to arrange into lamella along the fiber axis due to elongational flow and high stretching. Molecular orientation in the domains of the composite fibers was confirmed by polarized infrared spectroscopy. We demonstrated that formation of the oriented domains by electrospinning develops a hierarchical structure, which consequently modifies spectral properties because new multiple sharp lines appeared in the photoluminescence (PL) spectra of the fibers. In contrast to randomly deposited fibers, the PL intensity of uniaxially aligned fibers was found to be angle dependent. We propose that the elongated internal structure within the fibers controls the spontaneous emission of CdSe nanoparticles dispersed throughout the electrospun mat. A discussion on the nature of the controlled spontaneous emission is provided.
\end{abstract}

\section{Introduction}

Polymeric nanostructured materials with periodicity in structure and composition has led to a range of interesting and sometimes unique effects that offer potential and existing applications in photonics and optical data storage. ${ }^{1}$ The selfassembly of diblock copolymers, where two chemically different polymer blocks are joined covalently end-to-end, is one of the common approaches in formation of materials with periodic structures. ${ }^{2,3}$ Because of the difference in thermodynamic incompatibility of constituent monomers, respective blocks tend to phase separate and segregate into microdomains. ${ }^{4}$ In addition, segmented copolymers consisting of alternating comonomer segments also develop microphase separation under appropriate conditions. ${ }^{5}$ Polycarbonate urethane (PCU) is one of the versatile segmented copolymers that exhibits a two-phase microstructure where carbonate and urethane groups refer to soft segments and hard segments, respectively. The phase separation in the segmented copolymer arises from the packing of hard segments and/or their ability to establish strong secondary interactions such as H-bonding. ${ }^{6}$ Such microphase separation imparts various useful applications in terms of mechanical,${ }^{7}$ biomedical, ${ }^{8,9}$ and thermal properties. ${ }^{10}$ However, a few number of reports in the literature have addressed an optical feature of the segmented copolymers or systems they involve.

The ability to achieve a preferred orientation of microdomains in long range opens the door to the exploration of various applications associated with hierarchical structures of diblock/ segmented copolymers. ${ }^{11}$ Much interest in the field of copolymers has been focused on the ordering and orientation of the

* To whom correspondence should be addressed. Telephone: +90232 75075 11. Fax: +90 23275075 09. E-mail: mdemir@iyte.edu.tr and serdarozcelik@iyte.edu.tr. domain patterns by various means such as solvent vapor annealing, ${ }^{12}$ shear field, ${ }^{13}$ electrical field alignment,${ }^{14}$ magnetic field alignment, ${ }^{15}$ and thermal gradients. ${ }^{16}$ Electrospinning, known as a fabrication process of mesoscale diameter fibers from elongation of polymer solutions under high electrical fields, appears to be one of the promising techniques that allows orientation of copolymer domains. ${ }^{17-19}$ The nature of electrospinning is based on the generation of a continuously charged jet ejected from a polymer solution droplet to a grounded conductive plate. ${ }^{20,21}$ The mechanism of the process is mainly a result of the whipping of the fluid jet originating from the Coulombic interactions between the external electrical field and surface charges on the jet. Stretching and elongation of the unstable fluid jet result in the formation of fibers with micrometer to nanoscale diameters. In addition, an elongation rate on the order of $1000 \mathrm{~s}^{-1}$ is quite significant in terms of chain and domain orientation in electrospinning processes. ${ }^{22}$ Electrospun fibers are usually collected on sheetlike conductive electrodes as a nonwoven mat such that the fibers are randomly distributed with no preference to direction. In fact, they can be organized into uniaxially aligned arrays simply modifying the geometry of electrodes where fibers are collected ${ }^{23}$ For example, using a pair of conductive substrates separated by a void gap as the electrode allows the production of randomly deposited as well as uniaxially aligned fibers in a single run of electrospinning. The fibers deposited on parallel-positioned metal strips are randomly oriented, and the ones formed in an air gap are highly aligned normal to the edge of the metal strips.

Organization of the domains under a high electrical field ultimately offers a feasible tool to obtain structures positioned uniformly throughout the fibers. ${ }^{24-27}$ For example, preferential incorporation of nanoscale particles into one of the oriented 
domains within aligned fibers can lead to a hierarchical periodic structure in three dimensions. ${ }^{24,28-30}$ This method, in turn, can provide a template for novel materials with unprecedented optical features. Here, we describe loading of hydrophobically modied $\mathrm{CdSe}$ nanoparticles into segmented polycarbonate urethane (PCU) fibers and orientation of the internal domains along the fibers axis via electrospinning. CdSe nanocrystals are considered as one of the most promising emitting materials because their emisson color can be precisely tailored from blue to red. ${ }^{31}$ Structural formation of polymeric electrospun fibers in the presence of nanoparticles under elongational force leads to emergence of multiple sharp lines in the photoluminescence spectra that have not been reported so far in electrospinning literature. Moreover, we have observed that the emission feature of $\mathrm{CdSe}$ dispersed in fibers is strongly influenced by the alignment of fibers.

\section{Experimental Section}

Segmented PCU $\left(M_{\mathrm{n}} \sim 73000\right.$ and $M_{\mathrm{w}} \sim 237000$ by GPC) based on poly(1,6-hexyl-1,2-ethyl carbonate) diol, 4,4'-methylenebis(phenyl isocyanate) and 1,4-butanediol was provided from Sigma-Aldrich. The chemical structure of the copolymer is given in Figure S.1 of the Supporting Information. CdSe nanoparticles capped with tri- $n$-octylphosphine oxide (TOPO) were synthesized and characterized in our laboratory following the procedure by Pan et al. ${ }^{32}$ The desired amount of PCU was dissolved in dimethyl formamide (DMF) at room temperature. CdSe dispersion was added dropwise into a polymer solution followed by ultrasonication for $10 \mathrm{~min}$ while stirring. This precursor dispersion in a mixture of dimethylformamide and toluene was subjected subsequently to electrospinning at 1.5 $\mathrm{kV} \mathrm{cm}{ }^{-1}$. (applied voltage, $12 \mathrm{kV}$; spinning distance, $8 \mathrm{~cm}$ ) The fibers were collected on two parallel-positioned metal strips and on $\mathrm{Al}$ foil. Photoluminescence was measured with Varian Cary 50 spectrophotometer. Thermogravimetric and dynamical scanning calorimeter analyses were performed with a Perkin Elmer Diomand thermogravimetric/differential thermal analysis (TG/DTA) system under a nitrogen atmosphere with a step size of $10{ }^{\circ} \mathrm{C} \mathrm{sec}^{-1}$. The derivative thermogravimety (DTG) results were obtained by taking the temperature derivative of mass loss, $\mathrm{d}\left(w / w_{0}\right) / \mathrm{d} T$, at a constant heating rate, where $w$ is the sample weight and $w_{0}$ is the initial sample weight. The diameter of the fibers was determined by statistical treatment of the scanning electron microscopic (Phillips XL-30S FEG) images. Phase separation of the copolymer was observed with a Digital Instruments Multimode AFM with a silicon probe in air using the tapping mode. Transmission electron microscopy (TEM) was performed with a Zeiss 912 Omega microscope working at a voltage of $120 \mathrm{kV}$ and with a Technai F20 microscope working at a voltage of $200 \mathrm{kV}$ SFEG. Confocal microscopic images were captured with an Andor Revolution confocal microscope. Infrared spectra were obtained with a PerkinElmer Spectrum 100 Fourier transform infrared (FTIR) spectrometer with a spectral resolution of $4 \mathrm{~cm}^{-1}$. Polarization of the incident infrared radiation was achieved with a $\mathrm{ZnSe}$ polarizer.

\section{Results and Discussion}

CdSe nanoparticles were prepared ex situ in toluene and added into the copolymer solution dropwise under stirring prior to electrospinning. Figure 1 presents spectroscopic and structural properties of CdSe nanoparticles obtained by optical spectroscopy, X-ray diffraction, and TEM. In panel a, absorption and fluorescence spectra of the particles are given. The particles absorb at $505 \mathrm{~nm}$ and emit at $540 \mathrm{~nm}$. Photoluminescence with
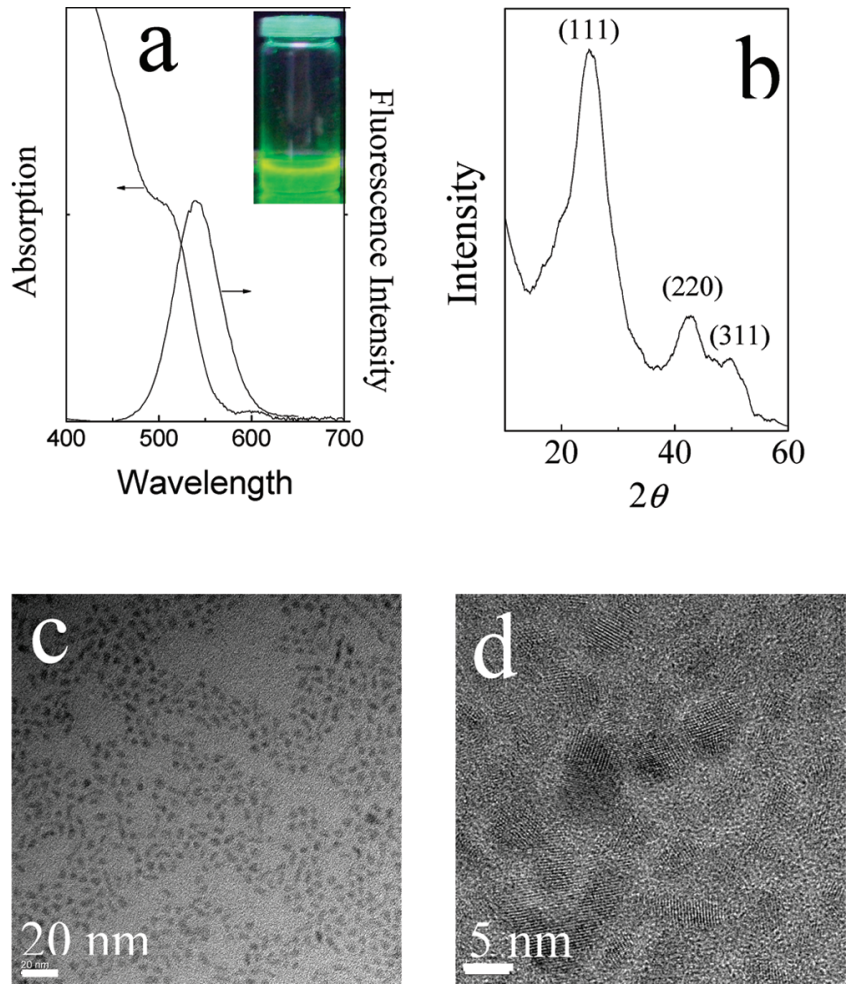

Figure 1. (a) Absorption and photoluminescence spectra of the CdSe particles. A photograph of the particle dispersion in toluene under UV light is presented in the inset. (b) X-ray diffractogram of as-synthesized CdSe indicating the crystalline features of the nanoparticles (JCPDS card 19-0191). (c) Overview of a TEM micrograph of the particles cast from toluene showing a uniform diameter of $5 \mathrm{~nm}$ with no aggregation. (d) High-resolution TEM micrograph of CdSe particles demonstrating lattice fringes.

a considerable efficiency (21\% quantum yield) has been observed. Although the dispersion of particles in toluene is colorless under daylight, they show strong green emission under UV light (inset of panel a). Panel b of Figure 1 demonstrates a typical XRD of zinc blend CdSe nanoparticles. Panel $\mathrm{c}$ and $\mathrm{d}$ show an overview and a high-resolution transmission image of an electron microscope (TEM) image of the CdSe particles, respectively. The particles shown in the images have a uniform size of $5 \mathrm{~nm}$ without aggregation. High-resolution images of the particles indicate that all particles are single crystalline.

Figure 2a presents thermogravimetric analysis of PCU with 10 wt $\%$ CdSe particles performed between room temperature and $700{ }^{\circ} \mathrm{C}$. The left and right axes display mass and derivative of mass, respectively. The data show two weight losses at different temperatures, which indicates that decomposition occurs at two distinct steps. The first step of the thermal decomposition is simple depolymerization of urethane bonds (75\% mass loss), and the second step is soft PC segment decomposition (15\% mass loss). The phase separation process of cast PCU film was confirmed by differential scanning calorimetry (DSC). Two glass transition points $\left(T_{\mathrm{g} 1}=-2{ }^{\circ} \mathrm{C}\right.$ and $T_{\mathrm{g} 2}=49{ }^{\circ} \mathrm{C}$ ) can be observed in the thermogram (Figure $2 \mathrm{~b}$ ) of the film attributed to soft and hard segments, respectively. These values are consistent with the literature. ${ }^{9}$ A bimodal sharp exotherm exists at 1.9 and $3.8^{\circ} \mathrm{C}$ associated with crystallization of the soft and hard segments, respectively. Figure $2 \mathrm{c}$ shows a tapping mode AFM phase image of a PCU/CdSe film prepared by casting from DMF solution. The topographic image of the surface (Figure S.2 of the Supporting Information) appears relatively uniform and does not show any surface structure 

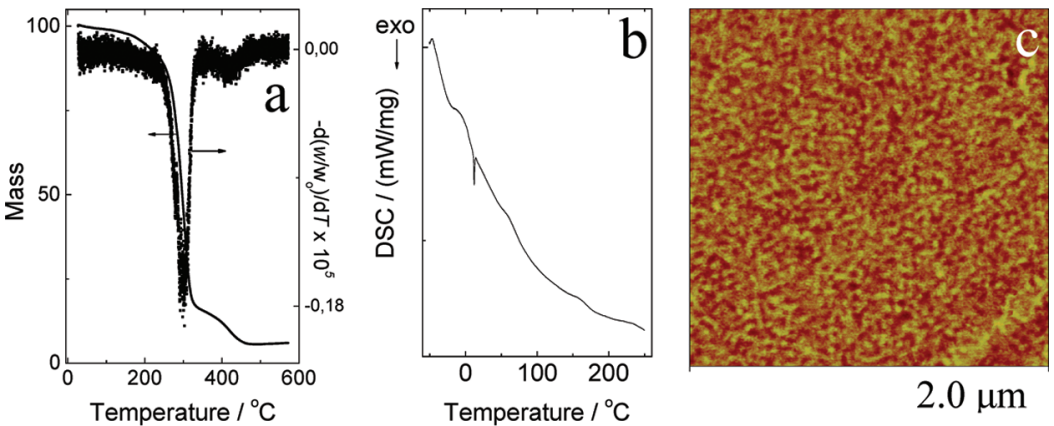

Figure 2. (a) Mass and derivative of mass of the PCU/CdSe fibers with TGA showing the composition of the monomers. (b) DSC thermogram of a PCU/CdSe composite film prepared by casting shows phase separation in bulk. (c) AFM tapping mode phase image of PCU film prepared by casting in the presence of $6 \mathrm{wt} \% \mathrm{CdSe}$ nanoparticles. The phase angle of the image is $20^{\circ}$.
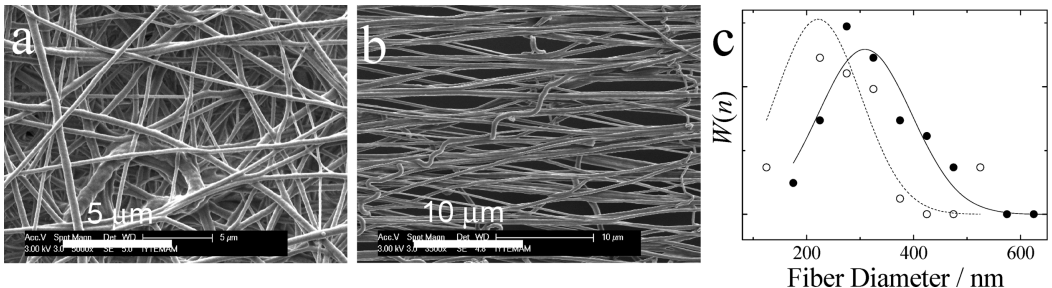

Figure 3. SEM images of (O) randomly deposited fibers on the metal strips and (O) uniaxially aligned fibers in the void gap prepared by electrospinning of $8 \mathrm{wt} \%$ dispersion at $1.5 \mathrm{kV} \mathrm{cm}^{-1}$. Diameter distributions of both fiber assemblies are shown in panel c.

feature; however, valuable information regarding phase separation is observed in phase imaging. In Figure 2c, the soft segments (carbonate-rich areas) give a dark contrast and the hard segments (urethane) appear bright, provided that the phase angle difference $\left(20^{\circ}\right)$ is due to hardness contrast. The hard segments aggregate into isolated domains that are randomly dispersed in the continuous matrix of the soft segments. The phase image indicates a morphology consisting of randomly oriented rodlike features whose dimensions are on the order of $100 \mathrm{~nm}$ in length and $20 \mathrm{~nm}$ in width on average. These spheres are most likely portions of rodlike domains that are standing normal to the substrate surface.

PCU solutions in DMF were subjected to electrospinning at $1.5 \mathrm{kV} \mathrm{cm}^{-1}$. Morphology of the fibers and their spatial distribution were examined by SEM. In a typical electrospinning setup, where a sheetlike grounded collector was employed, the electrical field is axially symmetric and provides no preference in direction of fibers, resulting in a nonwoven mat of randomly deposited fibers (Figure 3a). Replacing the sheetlike collectors with the one containing a void gap in its middle, for example, parallel metal strips, we directed the field lines toward the edge of the strips, and the charged fibers tend to locate between the electrodes. In this manner, highly aligned fibers were obtained in an area scaled with millimeters (Figure $3 b$ ). Results of the experiments showed that the level of alignment is significantly improved when the edge of the metal strip is sharpened, and therefore, the field lines are focused in the vicinity of the strips. Consequently, randomly deposited and uniaxially aligned fibers can be obtained via electrospinning. The diameters of the fibers were in the range of $100-500 \mathrm{~nm}$ in both cases for a given weight percent of the copolymer/nanoparticle dispersion. The geometrical shape of the randomly deposited and uniaxially aligned fibers did not appear to be different; however, the average diameter of the aligned fibers was found to be $\sim 100$ $\mathrm{nm}$ thinner than the randomly deposited fibers (Figure $3 \mathrm{c}$ ). This result was expected because the latter fibers were affected by additional stretching across the gap provided by the metal strips.

Figure 4 presents TEM images (panels a,b) and a fluorescent confocal microscope image (panel c) of PCU fibers with $12 \mathrm{wt}$
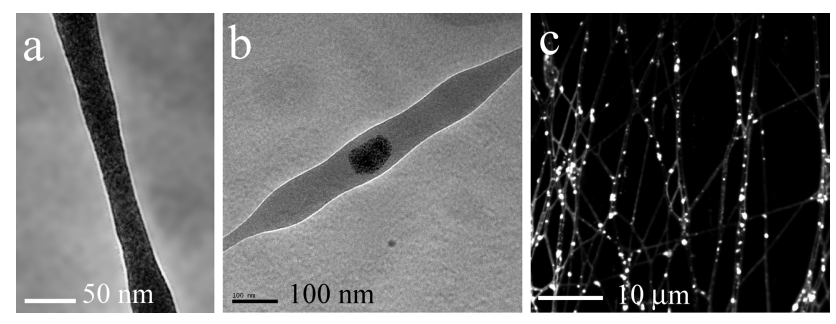

Figure 4. TEM (a,b) and florescence confocal microscope (c) images of electrospun PCU fibers with $12 \mathrm{wt} \%$ CdSe particles at different magnifications. Fibers are excited by an argon ion laser operating at $488 \mathrm{~nm}$ for confocal images.

$\%$ CdSe nanoparticles at different magnifications. The fibers used in spectroscopic analysis have a diameter of approximately $250 \mathrm{~nm}$. This specimen size is too thick for TEM analysis to resolve the location of the CdSe particles. Thus, the fiber diameter was reduced to $\sim 50 \mathrm{~nm}$ by dilution of the electrospinning dispersion. The CdSe nanoparticle surface was tailored to have a strong affinity to the copolymer. This feature was achieved by grafting TOPO molecules onto the surface with compact packing. This good compatibility of the CdSe particle surface and surrounding copolymer helps the embedment of the particles into the fibers, and the small size of the particles led to an even distribution along the thickness of the fibers (Figure 4a). However, a number of aggregates accumulated in a size of 50-100 nm were detected over the specimen (Figure 4b). The aggregates appear as a loose association of individual particles. Dilution of the electrospun dispersion for TEM analysis reduces viscosity and causes the appearance of axisymmetric instability in the fluid jet, which is a result of competing solution viscosity, surface tension of the electrospinning dispersion, and electrostatic interactions between a charged jet and an external electrical field. Therefore, the electrospinning process does not give rise to fibers with uniform diameters but rather to fibers displaying modulations of the diameter as the one shown in panel $b$ of Figure 4. The distribution of CdSe particles over the electrospun mat was confirmed by fluorescence confocal microscopy. A representative image in Figure 4c shows that 
the CdSe nanoparticles remain uniformly and selectively dispersed inside the fiber volume in spite of the high particle loading. The optical resolution of the confocal microscope is around $300 \mathrm{~nm}$. Therefore, a single nanoparticle with a diameter of $5 \mathrm{~nm}$ will appear as an emitting spot with a size of $300 \mathrm{~nm}$. The bright regions are fluorescent spots that may be a group of CdSe particles that are close to each other and/or aggregates of individual particles in the fibers. The number of particles in a fluorescent spot determines the brightness of the spot. Thus, the size of the particles observed in the fluorescence microscope image appears to be larger than those seen in TEM images.

The placement of particles with respect to copolymer domains has a strong influence on the macroscopic performance of copolymer/nanoparticle composites. ${ }^{28}$ This morphology is determined by entropic ${ }^{33}$ and entalpic ${ }^{34}$ effects. In the former, sizeselective organization of nanoparticles takes place. The ratio of the diameter of nanoparticles $d$ over domain spacing $L$ governs the location of particles in the composite structure. Theoretical studies suggest that smaller particles with a ratio $(d / L)$ of about 0.20 segregate at the interface of domains. ${ }^{33}$ In our particular case, the ratio of particle size over the size of domains is 0.12 , which is far below the given threshold value, so the particles are expected to be present at the interface of the domains. The latter effect involves the enthalpic interaction between the particle surface and copolymer blocks. If particles are compatible with one of the two domains, they favor the domain that is rich in terms of this segment more than the other. Here, the surface tensions of bulk PU and PC are $\gamma_{\mathrm{PU}} \sim 35 \mathrm{mN}$ $\mathrm{m}^{-135}$ and $\gamma_{\mathrm{PC}} \sim 43 \mathrm{mN} \mathrm{m}^{-1},{ }^{36}$ respectively. CdSe particles are densely covered by TOPO molecules, i.e., the particle surface is modified with aliphatic hydrocarbon chains whose surface energy $\left(\gamma_{\text {hydrocarbon }} \sim 30-33 \mathrm{mN} \mathrm{m}^{-1}\right)^{35}$ is closer to that of bulk PU. Therefore, CdSe nanoparticles are likely to be located in the urethane segment-rich phase according to enthalpic consideration. In both scenarios regarding the placement of particles in the fibers, CdSe nanoparticles are directed toward a defined spatial arrangement determined by phase morphology of the copolymer during electrospinning. This structural organization provides a unique medium that modifies emission of $\mathrm{CdSe}$ nanoparticles emphasizing structure-property relationships in hierarchically ordered composite fibers.

The phase separation of copolymer domains in long range usually occurs in general at a slow rate of solvent evaporation with long annealing time. However, electrospinning is a quite fast process. The total time in fiber formation is estimated to be approximately $0.2 \mathrm{s.}^{22}$ Because of rapid solvent evaporation and strong elongational flow to be on the order of $10^{5} \mathrm{~s}^{-1},{ }^{37}$ classical copolymer structures do not occur, rather the domains are distorted parallel to the direction of electrical force. The surface structure of electrospun PCU fibers were studied by tapping mode AFM. In a typical tapping mode AFM analysis, a cantilever oscillates at or near its resonant frequency during a scan of a surface of a flat specimen. Because the electrospun fibers have a cylindrical shape, the curvature of the surfaces of the fibers causes a vagueness from the center toward the edge of the fibers. To minimize the effect of curvature, thick fibers are produced to clarify phase separation on the surface of the fibers. Figure 5 shows phase images of the PCU fibers deposited on $\mathrm{Al}$ foil. Dashed lines in the magnified image (right panel) mark the borders of fibers. In contrast to cast film (Figure 2c), where extensive aggregation of hard segments take place in all directions, more developed and elongated surface structures were formed in electrospun fibers. This phase contrast on the surface of the electrospun fibers could be a result of alignment of CdSe particles or aggregates and/or aggregates of the

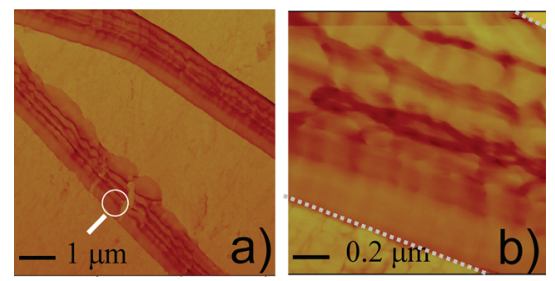

Figure 5. AFM phase image of electrospun fibers on $\mathrm{Al}$ foil prepared by electrospinning of $13 \mathrm{wt} \%$ PCU solution consisting of $6 \mathrm{wt} \% \mathrm{CdSe}$ particles with respect to $\mathrm{PCU}$ at $1.5 \mathrm{kV} \mathrm{cm}^{-1}$. Right panel is a magnified image, and the dashed lines mark the fiber boundaries. Phase angles of the images are $100^{\circ}$ and $200^{\circ}$.

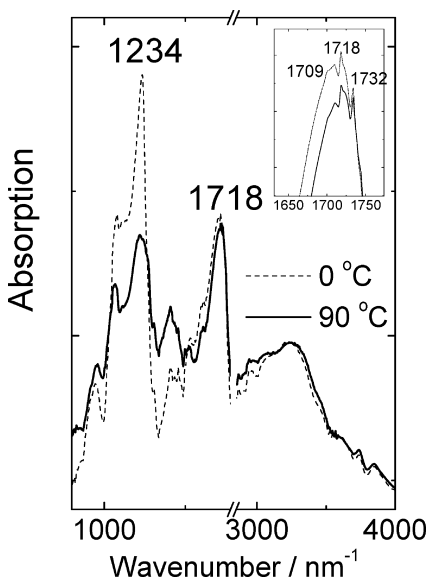

Figure 6. Polarized FTIR spectra for uniaxially aligned PCU fibers show that the peak intensity for polarized measurement parallel to the fiber axis is greater than that of the perpendicular measurement. In the inset, the frequency region, including carbonyl signals, is given in detail.

hard segment domains by electrospinning. The development of the aligned phase contrast is a distinct indication of strong deformation occurring on the surface of the fibers during the process. Similar distortion of copolymer domains upon electrospinning has been observed in bulk previously. ${ }^{18}$ Routsalainen et al. compared the phase of diblock copolymer structures evolved by annealing and electrospinning. The authors showed extended wormlike structures in electrospun fibers even if classical diblock structures are observed in the bulk state of polystyrene-block-poly(4-vinylpyridine).

The orientational order of the electrospun fibers investigated by vibrational spectroscopy can provide information at the molecular level. An infrared spectrum of uniaxially aligned PCU/CdSe fibers is shown in Figure 6. The main features involve the band at $1234 \mathrm{~cm}^{-1}$ and carbonyl stretch in the 1735-1699 $\mathrm{cm}^{-1}$ region. The former strong band can be assigned to $v(\mathrm{C}-\mathrm{N})+\delta(\mathrm{N}-\mathrm{H})$ vibrations. ${ }^{38,39}$ In the latter, the carbonyl stretching band between 1735 and $1699 \mathrm{~cm}^{-1}$ is split into three bands centered at 1732, 1718, and $1709 \mathrm{~cm}^{-1}$. They may be due to carbonyl stretchings within different secondary structures. ${ }^{40}$ The band at $1732 \mathrm{~cm}^{-1}$ can be typically assigned to free (non-hydrogen-bonded) urethane carbonyls. While the band at $1709 \mathrm{~cm}^{-1}$ may be assigned confidently to ordered, for example, hydrogen-bonded carbonyl, the intermediate frequency value of $1718 \mathrm{~cm}^{-1}$ allows for several alternative approaches such as associating the intermediate frequency value with irregularly hydrogen-bonded carbonyls, different H-bonding geometry at a frequency between free and ordered hydrogenbonded limits, and or to transition dipole-dipole coupling in a highly ordered structure. ${ }^{40}$ The ordered feature of hard segments detected by IR spectra was further investigated by SAXS to determine the maximal distance of hard segments. The length- 

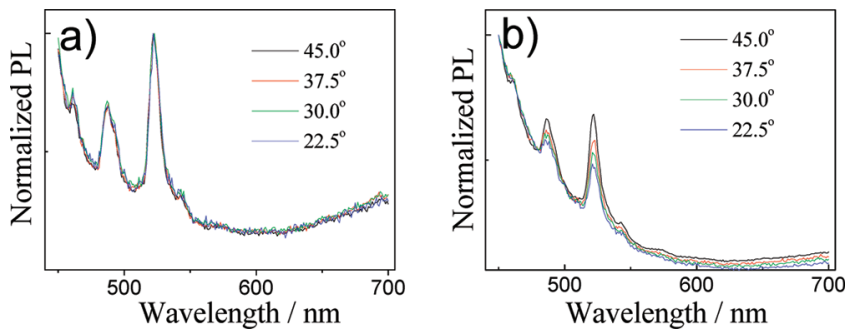

Figure 7. PL spectra of (a) randomly deposited and (b) uniaxially aligned fibers produced by electrospinning of $8 \mathrm{wt} \%$ solution at 1.5 $\mathrm{kV} \mathrm{cm} \mathrm{cm}^{-1}$. $\left(\lambda_{\mathrm{ex}}=380 \mathrm{~nm}\right)$.

scale covered in the measurements was in range of $2.9-29 \mathrm{~nm}$ $\left(0.15-1.5^{\circ}\right)$. However, the SAXS pattern of both samples (Figure S.3 of the Supporting Information) showed no clear signal, which suggests the sizes of the crystalline hard segments are out of the size range, if they exist.

Polarized FTIR was used to estimate the orientation behavior in segmented linear PCU. This method utilizes linearly polarized IR radiation to characterize the orientation of specific chromophoric groups of a polymer molecule. Therefore, it allows for separation of the orientation behavior of different segments in such multiphase materials. ${ }^{41}$ Randomly deposited and uniaxially aligned fiber assemblies were analyzed. Randomly deposited fibers exhibit almost identical IR spectra regardless of the polarization of the incident IR beam (not shown). However, when uniaxially aligned fibers were analyzed, the difference in the spectra was striking. No shift was observed in the positions of the bands; however, the intensity of absorption varies depending on the polarization state of the incident beam. In segmented PCU, the orientation of soft and hard segments can be represented by the bands of $v\left(\mathrm{CH}_{2}\right)$ at $2937 \mathrm{~cm}^{-1}$ and $v(\mathrm{C}-\mathrm{N})+\delta(\mathrm{N}-\mathrm{H})$ at $1234 \mathrm{~cm}^{-1}$ in the FTIR spectra of the copolymer, respectively. While the intensities of the former band are comparable at both polarization states, the latter showed a pronounced increase when the incident beam is polarized parallel to the uniaxially aligned fibers compared to when it is perpendicularly polarized. This result suggests higher levels of orientation for the hard segments than for the soft ones at the same level of deformation during the electrospinning process. Stress-induced ordering begins in the soft segments as a result of a decrease in entropy. ${ }^{7}$ Not surprisingly, soft segments tend to relax quickly and take on a more random orientation, whereas hard segments tend to retain their level of orientation upon deformation due to their rigidity. Consequently, hard segments exhibit a significant amount of orientation with the same elongation. These infrared dichroism experiments revealed the presence of segmental orientation in the elongation direction of individual nanofibers produced by electrospinning.

Spectroscopic properties of emitting species can be studied by photoluminescence spectroscopy. Angle-dependent photoluminescence is a tool to study effects of layered domains on emission properties of the nanoparticles. Figure 7 shows angleresolved photoluminescence (PL) spectra of CdSe nanoparticles dispersed in electrospun PCU fibers that are randomly oriented on the metals strips and uniaxially aligned within the space of the strips (SEM images, Figure 3). PL spectra of both types of fibers demonstrate similar spectral features. The PL intensity of randomly oriented fibers does not represent angle dependence; however, a variation in PL intensity of the aligned fibers as a function of angle is evident. A striking feature in both spectra is the appearance of discrete sharp emission lines at 460, 487, 522 , and $543 \mathrm{~nm}$. The full width at half-maximum (fwhm) of the most intense peak at $522 \mathrm{~nm}$ is $290 \mathrm{~cm}^{-1}(36 \mathrm{meV})$, yielding

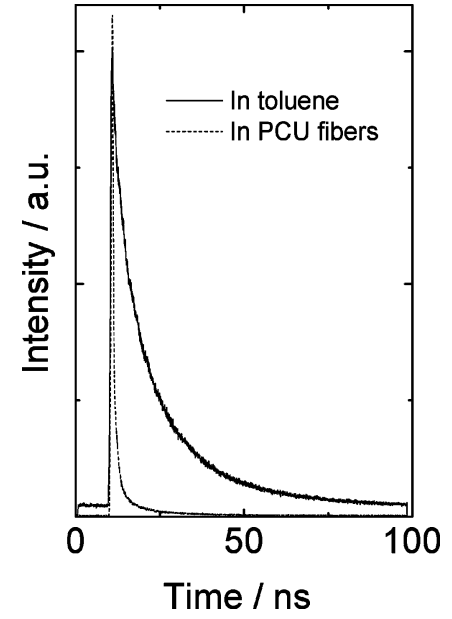

Figure 8. Fluorescence lifetime decays of CdSe particles in toluene and electrospun PCU fibers.

a 7-fold reduction in fwhm compared to that of the nanoparticles dispersed in toluene. A 6-fold reduction of fwhm for the peak at $487 \mathrm{~nm}$ is measured as well. CdSe nanoparticles in toluene emit at $540 \mathrm{~nm}$ with a fwhm of $2050 \mathrm{~cm}^{-1}(250 \mathrm{meV})$ (Figure 1a). As compared to nanoparticles dispersed in toluene, these discrete peaks at 522 and $487 \mathrm{~nm}$ are respectively shifted to higher energies of 640 and $2015 \mathrm{~cm}^{-1}$, respectively. The observed discrete and blue-shifted lines of the PL spectra of CdSe nanoparticles dispersed in PCU fibers lead us to consider that optical interference or microcavity effects may be operative.

Alignment of the fibers may cause a modulated PL intensity, as an indication of exciton-photon coupling within fibers. The coupling may arise from elongated internal morphology formed by a phase separation of soft and hard segments of the PCU because these segments possess a substantial difference in hardness confirmed by AFM observation (Figure 5). The fibers should provide a medium that may act as a cavity at nanometer scale and may cause a coupling of cavity modes to photons. Excitation wavelength dependence of PL was also studied to determine coupling of excitons to phonons. No change was observed in the spectral shape and position of the lines in the PL spectra, eliminating the possibility of exciton-phonon coupling. More information on exciton dynamics of nanoparticles is needed to gain further insights about exciton-photon coupling.

Fluorescence lifetime measurements may provide information regarding exciton dynamics. Fluorescence decay times of $\mathrm{CdSe}$ nanoparticles dispersed in toluene and in electrospun fibers are totally different. Average decay times of the nanoparticles were 9.80 and $0.08 \mathrm{~ns}$ in toluene and electrospun fibers, respectively (Figure 8). A 123-fold shortening in decay time indicates that exciton dynamics of $\mathrm{CdSe}$ nanoparticles are strongly modified in the electrospun fibers. The nature of this reduction is not understood and requires further systematic investigation. Meanwhile, a combination of a very strong modification of exciton dynamics, along with spectral modulation of CdSe emission in electrospun fibers, may point out interference effects.

The signatures of exciton-photon coupling are the modulation of spectral and spatial distributions of emission signals and modified exciton dynamics. ${ }^{42-44}$ Depending on the interaction strength (also known as Rabi splitting), two types of coupling exist: weak and strong. In weak coupling, spectral and spatial distributions of emission signals are modulated, but exciton dynamics are not perturbed. In contrast, strong coupling (mixing of exciton and photon states) initiates an anticrossing of spectral lines, establishes a Rabi splitting, and strongly modifies exciton 
dynamics. In our case, PL signatures (intensity, spectral position, and shape) are strongly modified in electrospun fibers, including variation in intensity, reduction in fwhm, formation of discrete lines, and blue shift of the discrete lines. When the spatial position of the aligned fibers is altered by rotating of the sample stage, PL intensity is further modulated. Moreover, a strong adjustment of exciton dynamics was observed. However, anticrossing and Rabi-splitting phenomena are not observed. Therefore, these observations partially support the existence of exciton-photon coupling in the fibers. As a control experiment, a cast film was prepared from the same PCU/CdSe dispersion that was subjected to electrospinning. No modified signals were detected in PL spectra in the films. This control experiment proves that the hierarchical structural organization of copolymer domains formed in electrospun fibers is crucial to control spontaneous emission of CdSe nanoparticles. Exciton-exciton interactions may also be considered to explain these experimental results, but it requires excitation intensity dependence to study optical input-output characteristics of the CdSe nanoparticles dispersed in toluene and electrospun fibers.

\section{Conclusion}

This paper outlines a methodology for producing emitting structures at optical-length scales using a combination of electrospinning and self-organization processes. Mesoscale diameter composite fibers of segmented polycarbonate urethane and CdSe nanoparticles have been prepared by electrospinning. Elongational flow of the copolymer/nanoparticle dispersion under a high electrical field reshapes the internal copolymer domains along the fibers. Consequently, structural organization of the domains within the fibers modifies spontaneous emission of CdSe nanoparticles that are evenly dispersed throughout the fiber volume. Controlling spontaneous emission by organization of copolymer molecules and nanosized semiconductor particles is very attractive for numerous potential applications in electronics, optoelectronics, and magnetic storage devices. Ongoing research efforts are being directed to resolve details of the modulation mechanism of spontaneous emission originating from the PCU/CdSe nanocomposite system.

Acknowledgment. The authors thank members of the Materials Research Center of IYTE and I. Lieberwirth of MPI-P for microscopy work. M.M.D. acknowledges financial support from The Scientific and Technological Research Council of Turkey (TUBITAK) career development project (TBAG107T795). S.O. thanks the State Planning Organization (DPT2003K120690-6) of the Turkish government for financial support.

Supporting Information Available: Chemical structure of polycarbonate urethane, AFM topography images, and SAXS patterns of the cast and electrospun spun films. This material is available free of charge via the Internet at http://pubs.acs.org.

\section{References and Notes}

(1) Paquet, C.; Kumacheva, E. Mater. Today 2008, 11, 48

(2) Forster, S.; Plantenberg, T. Angew. Chem., Int. Ed. 2002, 41, 689.

(3) Lodge, T. P. Macromol. Chem. Phys. 2003, 204, 265.

(4) Bita, I.; Yang, J. K. W.; Jung, Y. S.; Ross, C. A.; Thomas, E. L. Berggren, K. K. Science 2008, 321, 939.

(5) Yilgor, I.; Yilgor, E. Polym. Rev. 2007, 47, 487.

(6) Hernandez, R.; Weksler, J.; Padsalgikar, A.; Choi, T.; Angelo, E.; Lin, J. S.; Xu, L. C.; Siedlecki, C. A.; Runt, J. Macromolecules 2008, 41, 9767.
(7) Curgul, S.; Yilgor, I.; Yigor, E.; Erman, B.; Cakmak, M. Macromolecules 2004, 37, 8676 .

(8) Christenson, E. M.; Anderson, J. M.; Hiltner, A. J. Biomed. Mater. Res., Part A 2004, 70A, 245. 5508 .

(9) Guo, J. T.; Zhao, M. H.; Ti, Y.; Wang, B. J. Mater. Sci. 2007, 42,

(10) Eceiza, A.; Larranaga, M.; de la Caba, K.; Kortaberria, G.; Marieta, C.; Corcuera, M. A.; Mondragon, I. J. Appl. Polym. Sci. 2008, 108, 3092. (11) Smart, T.; Lomas, H.; Massignani, M.; Flores-Merino, M. V.; Perez, L. R.; Battaglia, G. Nano Today 2008, 3, 38 .

(12) Kimura, M.; Misner, M. J.; Xu, T.; Kim, S. H.; Russell, T. P. Langmuir 2003, 19, 9910.

(13) Almdal, K.; Koppi, K. A.; Bates, F. S. Macromolecules 1993, 26, 4058 .

(14) Amundson, K.; Helfand, E.; Davis, D. D.; Quan, X.; Patel, S. S.; Smith, S. D. Macromolecules 1991, 24, 6546.

(15) Osuji, C.; Ferreira, P. J.; Mao, G. P.; Ober, C. K.; Vander Sande, J. B.; Thomas, E. L. Macromolecules 2004, 37, 9903.

(16) Bodycomb, J.; Hara, M. Macromolecules 1995, 28, 8190.

(17) Ruotsalainen, T.; Turku, J.; Heikkila, P.; Ruokolainen, J.; Nykanen, A.; Laitinen, T.; Torkkeli, M.; Serimaa, R.; ten Brinke, G.; Harlin, A.; Ikkala, O. Adv. Mater. 2005, 17, 1048 .

(18) Ruotsalainen, T.; Turku, J.; Hiekkataipale, P.; Vainio, U.; Serimaa, R.; ten Brinke, G.; Harlin, A.; Ruokolainen, J.; Ikkala, O. Soft Matter 2007, 3, 978.

(19) Kalra, V.; Kakad, P. A.; Mendez, S.; Ivannikov, T.; Kamperman, M.; Joo, Y. L. Macromolecules 2006, 39, 5453.

(20) Doshi, J.; Reneker, D. H. J. Electrost. 1995, 35, 151.

(21) Greiner, A.; Wendorff, J. H. Angew. Chem., Int. Ed. 2007, 46, 5670.

(22) Greiner, A.; Wendorff, J. H. Functional Self-Assembled Nanofibers by Electrospinning. In Self-Assembled Nanomaterials I, Advances in Polymer Science; Shimuzi, T. Ed.; Springer-Verlag: Berlin Heidelberg, 2008; Vol. 219; pp 107.

(23) Li, D.; Wang, Y. L.; Xia, Y. N. Adv. Mater. 2004, 16, 361.

(24) Kalra, V.; Lee, J.; Lee, J. H.; Lee, S. G.; Marquez, M.; Wiesner, U.; Joo, Y. L. Small 2008, 4, 2067.

(25) Demir, M. M.; Yilgor, I.; Yilgor, E.; Erman, B. Polymer 2002, 43, 3303.

(26) Demir, M. M.; Gulgun, M. A.; Menceloglu, Y. Z.; Erman, B.; Abramchuk, S. S.; Makhaeva, E. E.; Khokhlov, A. R.; Matveeva, V. G.; Sulman, M. G. Macromolecules 2004, 37, 1787.

(27) Demir, M. M.; Ugur, G.; Gulgun, M. A.; Menceloglu, Y. Z. Macromol. Chem. Phys. 2008, 209, 508.

(28) Bockstaller, M. R.; Mickiewicz, R. A.; Thomas, E. L. Adv. Mater. 2005, 17, 1331.

(29) Lin, Y.; Boker, A.; He, J. B.; Sill, K.; Xiang, H. Q.; Abetz, C.; Li, X. F.; Wang, J.; Emrick, T.; Long, S.; Wang, Q.; Balazs, A.; Russell, T. P. Nature 2005, 434, 55 .

(30) Goda, H.; Frank, C. W. Chem. Mater. 2001, 13, 2783.

(31) Talapin, D. V.; Mekis, I.; Gotzinger, S.; Kornowski, A.; Benson, O.; Weller, H. J. Phys. Chem. B 2004, 108, 18826.

(32) Pan, D. C.; Wang, Q.; Jiang, S. C.; Ji, X. L.; An, L. J. Adv. Mater. 2005, 17, 176 .

(33) Thompson, R. B.; Ginzburg, V. V.; Matsen, M. W.; Balazs, A. C. Science 2001, 292, 2469.

(34) Bockstaller, M. R.; Lapetnikov, Y.; Margel, S.; Thomas, E. L. J. Am. Chem. Soc. 2003, 125, 5276.

(35) Brandrup, J.; Immergut, E. H. Polymer Handbook, 3rd ed.; John Wiley \& Sons: New York, 1998.

(36) Madkour, T. M. Polycarbonate. In Polymer Data Handbook; Mark, J. E., Ed.; Oxford University Press: Cincinnati, 1999; pp 363.

(37) Reneker, D. H.; Yarin, A. L.; Fong, H.; Koombhongse, S. J. Appl. Phys. 2000, 87, 4531 .

(38) Wang, H. C.; Aubuchon, S. R.; Thompson, D. G.; Osborn, J. C.; Marsh, A. L.; Nichols, W. R.; Schoonover, J. R.; Palmer, R. A. Macromolecules 2002, 35, 8794.

(39) Bonini, C.; D’Auria, M.; Ernanuele, L.; Ferri, R.; Pucciariello, R.; Sabia, A. R. J. Appl. Polym. Sci. 2005, 98, 1451.

(40) Pollack, S. K.; Shen, D. Y.; Hsu, S. L.; Wang, Q.; Stidham, H. D. Macromolecules 1989, 22, 551.

(41) Seymour, R. W.; Allegrez.Ae; Cooper, S. L. Macromolecules 1973, 6,896 .

(42) Lidzey, D. G.; Bradley, D. D. C.; Pate, M. A.; David, J. P. R.; Whittaker, D. M.; Fisher, T. A.; Skolnick, M. S. Appl. Phys. Lett. 1997, $71,744$.

(43) Lidzey, D. G.; Bradley, D. D. C.; Skolnick, M. S.; Virgili, T.; Walker, S.; Whittaker, D. M. Nature 1998, 395, 53.

(44) Weisbuch, C.; Nishioka, M.; Ishikawa, A.; Arakawa, Y. Phys. Rev. Lett. 1992, 69, 3314.

JP903899S 